\title{
Similarities and Differences between China and EU in Similar Cases Shanshan Su${ }^{1, a *}$, Lanfang Fei ${ }^{1, b}$ \\ ${ }^{1}$ School of law / Intellectual Property Institute, Jinan University, Guangzhou, Guangdong, China \\ a445300062@qq.com, bfei27@ hotmail.com \\ ${ }^{*}$ Corresponding author
}

Keywords: horizontal monopoly agreement; overcapacity; Similarities and differences; Shanxi Electricity Case; Synthetic Fibers Case

\begin{abstract}
This article selects two cases, namely the first case of Shanxi Electricity Anti-monopoly Law Enforcement (hereinafter referred to as "Shanxi Electricity Case") and the EU Commission of Synthetic Fibers (hereinafter referred to as "Synthetic Fibers Case"). Both of these cases involve horizontal monopoly agreements and defenses of overcapacity. The following two cases will be analyzed and compared.This article will analyze and compare the two cases, comparing the similarities and differences between the two law enforcement decisions on the facts of the case, the main points of the referee, the defense opinions of both parties and the conclusion of the judgment, analyzing the reasons for the similarities and differences, and providing references for the handling of similar cases in the future.
\end{abstract}

\section{中欧类似案件异同解析}

一山西电力案与合成纤维案

\author{
苏珊珊 $1, a,{ }^{*}$ ，费兰芳 ${ }^{2, b}$ \\ 1暨南大学法学院/知识产权学院, 广州, 广东, 中国 \\ 2暨南大学法学院/知识产权学院, 广州, 广东, 中国 \\ a445300062@qq.com, bfei27@ hotmail.com \\ *苏珊珊
}

关键词：横向垄断协议; 产能过剩; 异同点; 山西电力案; 合成纤维案

中文摘要. 本文选取两个案例, 分别是中国的山西电力反垄断执法第一案（以下简称 “山西 电力案” ) 和欧盟的欧盟委员会合成纤维案（以下简称 “合成纤维案”）。这两个案例都涉 及到横向垄断协议和产能过剩的抗辩。本文将对两个案例进行分析和对比, 从案件事实、裁 判要点、双方抗辩观点和裁判结论上比较两个执法决定的异同, 剖析异同产生的原因, 为中 国未来类似案件的处理提供借鉴。

\section{1. “山西电力案” 案情简介}

2016年1月14日，山西省电力协会（以下简称 “山西电协”）召集23家发电企业，在太 原市召开火电企业大用户直供座谈会, 共同协商直供电交易价格, 会议签署通过了《山西省 火电企业防止恶意竞争保障行业健康可持续发展公约》（以下简称《公约》）。《公约》确定 
2016年山西省第二批大用户直供电报价较上网标杆电价让利幅度不超过 0.02 元/千瓦时, 最 低交易报价为 0.30 元/千瓦时。国家发改委依据《中华人民共和国反垄断法》13条的规定， 认为山西电协和 23 家涉案企业达成并实施了直供电价格垄断协议。

2017年8月3日，国家发改委指导山西发改委发布处罚决定：对达成垄断协议发挥组织作 用的山西电协顶格罚款 50 万元，对包括6家央企在内的涉案电力企业合计罚款 7338 万元。

\section{2. “合成纤维案” 案情简介}

1982年，占西欧合成纤维总产量 $70 \%$ ，占欧共体总产量 $85 \%$ 的合成纤维行业达成了一项 横向产量限制协议。协议针对的产品主要是聚酯胺纺织纱线、涤纶纺织纱线等合成纺织纤维。 达成产量限制协议的主要原因是需求疲软和产能过剩。协议的最主要内容是要求把合成纺织 纤维的产量限制在现有规模的85\%左右，使1986年的销售量稳定在1981年的水平。

1984年，欧共体委员会根据《欧共体条约》（现《欧盟运行条约》101条第3款）的豁免 规定批准了合成纤维行业的横向产量限制协议。

\section{3. 案件事实异同点}

\section{1 定性上:}

相同点:

两个案例都属于横向垄断协议, 都是处于同一市场水平上的具有竞争关系的企业达成的 有关限制市场竞争的协议。二者都属于 “核心卡特尔”，是最严重的竞争违法行为。

不同点:

横向垄断协议又可以细分为: 价格固定、产量限制、分割市场、联合抵制等具体类型。 山西电力案中经营者达成的协议属于横向的价格固定协议, 合成纤维案中经营者达成的协议 属于横向产量限制协议。

横向价格固定是指企业之间通过固定价格协议或协调各自的价格行为，达到获取垄断利 润、限制公平竞争的目的。在山西电力案中, 《公约》确定的让利幅度和最低交易报价是一 种明显的固定价格的行为。

横向产量限制是指：在市场经营过程中, 具有竞争关系的经营者之间, 相互约定限制商品 的生产数量的行为。在合成纤维案中, 合成纤维行业达成的协议的主要内容是要求把合成纺 织纤维的产量限制在现有规模的85\%左右, 使1986年的销售量稳定在1981年的水平。1981年 签约各方产品的销售总额为 164 万吨, 按照现有规模的 $85 \%$ 计算, 协议要求在 1985 年底前, 签 约各方的综合生产能力要削减总共 35.4 万吨。这是一种明显的产量限制的行为。

\section{2 产能过剩抗辩}

在两案中, 达成垄断协议的经营者均提出了产能过剩的抗辩。山西电力案中, 山西省电 力协会和涉案企业的一个重要抗辩理由是”电力行业经济不景气, 产能过剩。”合成纤维案 中, 从20世纪70年代开始, 合成纤维行业就存在”供需不平衡, 价格持续走低, 产能过剩。” 的问题，在这种背景下，合成纤维行业达成了产量限制的协议。

我国《反垄断法》第15条和《欧盟运行条约》101条第3款规定了横向垄断协议的抗辩是 由。中国和欧盟关于抗辩是由的具体规定虽不尽相同，但都包含了两个最关键的要素：1、 协议不会严重限制相关市场的竞争或有利于相关市场的竞争; 2、能使消费者分享由此产生 的收益。 
然而, 我国《反垄断法》15条是一种列举加概括式的规定, 其中第（五）项将产能过剩 明确为一种抗辩是由, 《欧盟运行条约》101条第 3 款是一种概括式的规定。关于产能过剩抗 辩在两个案件中的具体适用, 将在本文第五部分展开分析, 这里不再赘述。

山西电力案中，执法机构认为产能过剩抗辩不能成立，《公约》属于价格垄断协议; 而 在合成纤维中, 欧共体委员会认为抗辩成立, 依据《欧盟运行条约》101条第3款豁免了该项 横向产量限制协议。

\section{4. 比较}

中国从整体上借鉴了欧盟的反垄断执法模式。中国和欧盟均采取行政执法模式: 行政机 关负责反垄断法的实施和执行, 其既有权对涉嫌垄断的案件进行调查和审理, 又有权对案件 作出裁决, 行政机关在反垄断执法过程中既是调查者又是裁判者。在执法依据上均采取列举 加豁免模式。在执法程序上步骤类似。下面主要从执法依据, 执法机构, 执法程序三个方面 加以介绍。

\section{1 执法依据}

欧盟竞争法主要有以下三个渊源：1、属于一级立法规范的《欧共体条约》（现为《欧 盟运行条约》）；2、属于二级或三级立法规范的欧盟委员会或欧盟部长理事会制定的规范 性文件；3、欧洲法院的判决和判例。《欧盟运行条约》101条规定横向垄断协议也即卡特尔 协议, 第1款采用概括式列举方式, 先整体概括性阐述卡特尔协议再列举了 5 项常见的或者说 典型的卡特尔协议类型; 第3款规定了由四个条件共同组成的抗辩是由, 其中包括两个积极 条件: (1)有利于改善产品的生产或销售, 或有利于促进技术或经济进步; (2)消费者能公平分 享由此产生的利益; 和两个消极条件: (3)不对企业施加对这些目标的实现并非必不可少的限 制; (4)不会致使企业有可能在相关产品市场的重要部分消除竞争。在实践中, 欧盟卡特尔协 议的个案豁免要同时满足这四个条件。

中国的反垄断执法依据主要是《反垄断法》以及国务院和三大反垄断执法机构颁布的一 些配套规定。我国《反垄断法》对垄断协议的规定与欧盟有一定区别。首先, 我国将垄断协 议分为横向垄断协议 ( 《反垄断法》13条) 与纵向垄断协议 ( 《反垄断法》14条) ; 《欧盟 运行条约》101条第一款则包括了所有的垄断协议。

其次，《反垄断法》第13条采用的是列举加䚁底的模式，第1款列举了5项常见的横向垄 断协议类型，第6项为䛜底条款即反垄断执法机构认定的其他垄断协议，这与 101 条第 1 款的 概括式列举模式也不尽相同。

最后，我国《反垄断法》 15 条将垄断协议的豁免是由分为两个部分： 1 、属于第 1 款 $1-5$ 项的, 经营者要举证证明三项内容: (1) 即属于1-5情形中的一种; (2) 不会严重限制相关 市场的竞争; (3) 能够使消费者分享由此产生的利益。2、属于第1款6-7项的, 经营者仅需 证明一项内容, 即其垄断协议属于第6项或第7项规定的情形。而《欧盟运行条约》101条第3 款规定的适用豁免的四个条件统一适用于所有的垄断协议。

在举证责任的分配上, 《欧盟运行条约》101条对于横向垄断协议的规制设定了一个二 分结构的分析方法: 首先, 执法主体依第 1 款的规定负举证责任, 证明经营者达成或实施了 限制竞争的协议或行为; 其次, 在执法主体完成证明责任的基础上, 由经营者依第3款的规 定负证明责任, 证明其行为符合抗辩是由规定的条件从而免责。我国《反垄断法》在举证责 任的分配上的规定与《欧盟运行条约》相同, 执法机构依据《反垄断法》13条认定经营者达 成或实施了垄断协议, 经营者依据第 15 条对自己的行为进行抗辩时须举证证明其行为符合豁 免是由的相关规定。 


\section{2 执法机构}

欧盟方面, 反垄断法的执法机构是欧盟委员会, 欧盟委员会下设的竞争事务总司和竞争 事务专员, 具体负责欧盟反垄断法的执行。法院在欧盟反垄断法的执行过程中也发挥重要的 补充作用。当经营者不服欧盟委员会的处罚决定时, 可以通过向法院提起诉讼的方式来维护 自己的合法权益。随着时代的发展, 欧盟委员会的一部分反垄断执法权被不断稀释和分化, 或移交给其成员国的相关执法机构，或移交给法院。

我国目前的反垄断执法机构呈现出商务部、国家发改委、国家工商总局三足鼎立的局面, 并由国务院设立的反垄断委员会统一领导。我国《反垄断法》第 9 条、第 10 条明确规定, 反 垄断委员会负责组织、协调、指导反垄断工作, 并且可授权省级政府相关机构负责反垄断执 法工作。反垄断委员会领导三个执法机构: 一是商务部下设的反垄断局, 负责审查经营者集 中行为; 二是国家发改委下设的价格监督检查司, 负责依法查处价格垄断协议行为; 三是国 家工商总局下设的反垄断与不正当竞争执法局, 负责除经营者集中和价格垄断协议外的其他 反垄断执法工作。在实践中, 反垄断委员会只有组织协调功能, 反垄断执法工作由三机构分 工负责。

\section{3 执法程序}

在执法程序方面, 中国和欧盟都大致分为: 立案、初始评估、正式调查、处理决定这四 个方面, 其内容基本一致, 仅在一些程序性事项上或者对当事人权利的保障上有一些区别。 例如, 依据我国《反垄断法》的规定, 在正式调查阶段, 反垄断执法机关有权询问被调查的 经营者等相关人员, 并要求相关人员如实回答与调查有关的问题。而在欧盟的执法程序中, 被询问人有 “不得自证其罪” 的法定权利，可以拒绝回答执法机构的问题。

\section{5. 产能过剩抗辩}

由前面的分析可知, 在欧盟, 适用产能过剩抗辩需要同时满足四个条件, 在中国, 适用 产能过剩需要同时满足三个条件。接下来将结合案情进行具体分析。

在合成纤维案中, 欧盟委员会认为产能过剩抗辩成立, 批准了横向产量限制协议。主要 基于以下四个方面的理由:

首先必须考虑到1982年合成纤维行业存在的产能过剩的背景，产能过剩的原因是技术的 快速进步和需求疲软。

其次, 协议的目的是减少产能, 提高技术效率, 开发出更优质的产品。最终的结果应该 是提高盈利能力, 恢复各方的竞争力。据统计，1983年欧洲合成纤维行业的损失总额从1981 年的 12 亿德国马克, 降至5亿德国马克。因此, 协议有利于生产的提高和技术的进步, 并可 以促进经济的发展。第3条款要求协议为消费者提供所得利益的公平份额。在这种情况下, 最终形成的合成纤维行业将会更加健康, 更有竞争力, 为消费者提供更好的产品。

再次，该协议对当时的合成纤维产业来说是不可或缺的。市场已不能解决产能过剩问题 并维持一个有效的竞争关系。该协议仅涉及减少过剩产能，且持续时间有限。

最后，该协议对市场竞争的影响。是否阻碍竞争必须综合考虑市场特征，协议期限及其 中的规定。签约方不是市场上协议所涵盖产品的唯一供应商。许多其他生产商, 例如北美公 司等生产商也在市场上运作，与签约方竞争激烈。

欧盟委员会基于101条第3款所要求的四个条件对合成纤维行业情况进行综合分析，在合 成纤维行业同时满足四个条件的情况下，认定产能过剩的抗辩成立。

在山西电力案中, 适用产能过剩抗辩须同时满足三个条件: 首先是经济不景气导致生产 过剩的事实; 其次是达成的协议没有限制相关市场的竞争; 最后是消费者能共享收益。山西 
发改委未公开其行政处罚决定书, 仅公布了处罚决定。但依据这三个条件并结合本案案情分 析可知, 本案事实并不符合这三个条件:

首先，山西电力行业的产能过剩不是由于经济不景气的原因导致，而是由于电力行业的 市场化改革。从2013年起, 山西作为试点省份开始了大用户直供电交易, 鼓励大型工业用户 向发电企业直接购电、交易双方协商定价。在这种情况下，需要通过市场化、法治化的手段 有效推进火电行业供给侧结构性改革, 应充分发挥市场调节作用, 通过竞争方式实现优胜劣 汰, 缓解产能过剩, 实现供需平衡。

其次, 本次涉案经营者几乎涵盖了山西所有的发电企业。山西省电力协会组织发电企业 达成并实施了横向价格固定协议, 违背自由竞争原则, 其行为严重排除、限制了山西直供电 市场的公平竞争。

最后，消费者的合法权益受到损害。涉案企业实施了垄断协议，使得2016年山西第二批 大用户直接交易价格相较于 2016 年第一批有明显提升, 增加了下游实体企业的用电负担, 损 害了消费者利益。基于以上分析, 山西电力案经营者提出的抗辩不能成立。

通过以上分析我们可以看出，我国在反垄断执法上应更加注重信息公开，案件处理过程 和结果应更加公开透明。在执法决定中应更深入的分析和阐述事实认定和法律适用的说理部 分, 使执法决定更加完整, 说服力更强。这样才能更好地指导经营者在市场中的经营行为, 维护公平竞争的市场秩序，促进社会主义市场经济健康发展。

\section{致谢}

本文为国家自然科学青年基金一般项目《反垄断执法事后经济效果评估》(71603099)的 阶段性成果之一。

\section{References}

[1] Hou Dehong, Series the European Union antitrust enforcement. Science and technology and enterprise, 2013 (9).

[2] Yang Tao, Comparative study on enforcement mechanism of anti-monopoly law in China and foreign countries. Fudan university, 2013.

[3] Hou Dehong, Analysis of the implementation of the eu anti-monopoly law and its reference to China. Journal of heilongjiang political and legal management cadre college, 2013(03).

[4] Liu Xu, Perfection of Antitrust Committee's Function and Reform of Antitrust Law Enforcement Mechanism under the Background of Majority Reform: References from European Union and Germany . Economic Law Review, 2013, 24 (01).

[5] Pan Chong, On the Different Systems of Antitrust Law in the United States and Europe .Political Research Journal, 2014 (15).

[6] Pan Xinfa, Study on the exemption system of monopoly agreement. East China university of political science and law, 2008.

[7] Gao Junjie and Shen Jiao, Comparative analysis of the exemption system of American and European Union monopoly agreement and its reference significance. Journal of Yan 'an university (social science edition), 2008(01).

[8] $\mathrm{Wu}$ Dongmei, Economic analysis and legal application of horizontal price monopoly agreements: A case study of Hainan aerated brick price monopoly. China Price Regulation and Antitrust, 2014 (09). 